\title{
Wave Propagation in Oscillators Coupled by Time-Varying Resistor with Timing Mismatch
}

\author{
Yoko Uwate and Yoshifumi Nishio \\ Dept. of Electrical and Electronic Engineering, Tokushima University \\ 2-1 Minami-Josanjima, Tokushima, Japan \\ Email: \{uwate,nishio\}@ee.tokushima-u.ac.jp
}

\begin{abstract}
Synchronization phenomena in coupled oscillatory systems are very important model to describe various higherdimensional nonlinear phenomena in the field of natural science. In this study, we investigate synchronization phenomena in van der Pol oscillators coupled by time-varying resistors with timing mismatch as a ring. By carrying out computer simulations, we confirm the various interesting phenomena (wave propagation, clustering, complex phase pattern) which cannot be observed in simple oscillatory systems coupled by resistors.
\end{abstract}

\section{INTRODUCTION}

In recent years, many people have been trying to develop some applications to information processing by exploiting synchronization phenomena in coupled oscillatory systems. Such oscillatory systems can produce some kinds of phase patterns, and they may be utilized modeling of associative memory and learning process. Endo et al. have reported details of theoretical analysis and circuit experiments about some coupled oscillators as a ladder, a ring and a two-dimensional array [1]. Yamauchi et al. have discovered very interesting wave propagation phenomena of phase states between two adjacent oscillators in an array of van der Pol oscillators coupled by inductors [2].

On the other hand, there are some systems whose dissipation factors vary with time, for example, under the time-variation of the ambient temperature, an equation describing an object moving in a space with some friction and an equation governing a circuit with a resistor whose temperature coefficient is sensitive such as thermistor. However, there are few discussion about coupled oscillators coupling by a time-varying resistor.

In our previous research, we have investigated the synchronization phenomena in van der Pol oscillators coupled by timevarying resistor as a ring [3]. We confirmed the coexistence of in-phase and anti-phase synchronization and wave propagation phenomena. However, it is not make clear the mechanism of wave propagation in detail.

In this study, we investigate the wave propagation phenomena when timing mismatch is added to time-varying resistors. We realize the time-varying resistor by switching a positive and a negative resistor periodically. This proposed oscillatory system is novelty, this is because the coupling term has dynamics by switching value of resistor. First, the case of even number coupling, the coexistence of in-phase and anti-phase states are observed. In contrast, the case of odd number coupling, we can confirm the coexistence between in-phase and $n$-phase states. Second, the coexistence area with changing nonlinearity of oscillator is investigated. By carrying out computer simulations, we confirm the various interesting phenomena (wave propagation, clustering, complex phase pattern) which cannot be observed in simple oscillatory systems coupled by a resistor. Next, we carry out computer simulations when the timing mismatch is added to the TVR. The influence of small error for whole coupled system is investigated. We confirm that the different synchronization phenomena from the original coupled oscillatory system. For example, wave propagation disappear and wave extinction changes to wave propagation.

\section{Coupled Oscillators Model}

In this study, we consider a ring of oscillators as shown in Fig. 1. In this circuit adjacent two oscillators are coupled by one time-varying resistor (TVR). We realize the TVR by switching a positive and a negative resistors periodically as shown in Fig. 2. We introduce small resistors $r_{m}$ in computer simulations to avoid $L$-loop.

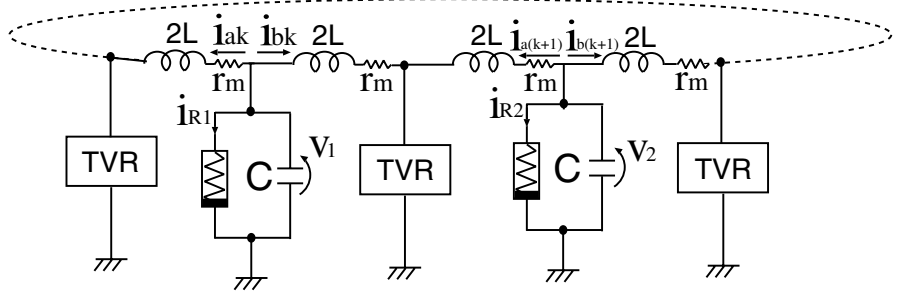

Fig. 1. Coupled oscillators model.

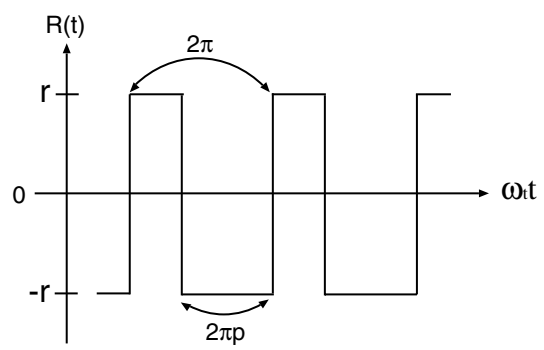

Fig. 2. Characteristics of the TVR.

First, the $v_{k}-i_{R k}$ characteristics of the nonlinear resistor are defined as follows,

$$
i_{R k}=-g_{1} v_{k}+g_{3} v_{k}^{3} \text {. }
$$

By changing the variables and the parameters,

$$
\begin{gathered}
v_{k}=\sqrt{\frac{g_{1}}{g_{3}}} x_{k}, \quad i_{k}=\sqrt{\frac{g_{1}}{3 g_{3}}} \sqrt{\frac{C}{L}} y_{k}, \quad t=\sqrt{L C} \tau, \\
\varepsilon=g_{1} \sqrt{\frac{L}{C}}, \quad \gamma=r \sqrt{\frac{C}{L}}, \quad \omega=\frac{1}{\sqrt{L C}} \omega_{t}, \quad \eta=r_{m} \sqrt{\frac{C}{L}},
\end{gathered}
$$


the normalized circuit equations of the array of oscillators are given as

$$
\left\{\begin{aligned}
& \frac{d x_{k}}{d \tau}=\varepsilon\left(1-x_{k}^{2}\right)-\left(y_{a k}+y_{b k}\right) \\
& \frac{d y_{a k}}{d \tau}=\frac{1}{2} x_{k}-\eta y_{a k}-\gamma(\tau)\left(y_{a k)}+y_{b(k+1)}\right) \\
& \frac{d y_{b k}}{d \tau}=\frac{1}{2} x_{k}-\eta y_{b k}-\gamma(\tau)\left(y_{a(k-1)}+y_{b k)}\right) \\
&(k=1,2, \cdots, N)
\end{aligned}\right.
$$

where

$$
y_{a 0}=y_{a N}, \quad y_{b(N+1)}=y_{b 1} .
$$

It should be noted that $\gamma(\tau)$ corresponds to the coupling strength and that $\varepsilon$ corresponds to the nonlinearity of oscillators. Eq. (2) is calculated by using the fourth-order RungeKutta method.

\section{SYNCHRONIZATION PHENOMENA}

\section{A. Even Number Coupling: $N=14$}

Figure 3 shows the computer simulated result for the case of $N=14$. $N$ denotes the number of coupled oscillators. We can see that the array of oscillators coupled by TVR are synchronized at in-phase or at anti-phase.

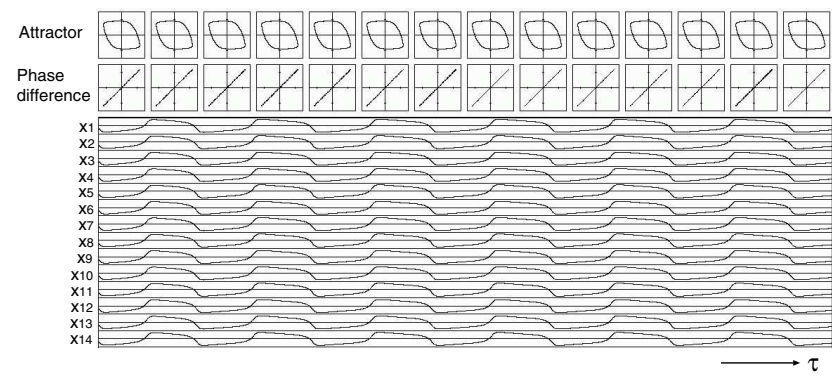

(a) In-phase synchronization.

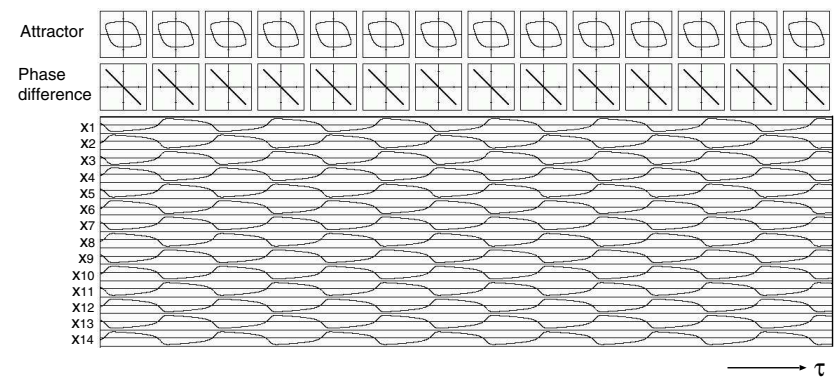

(b) Anti-phase synchronization.

Fig. 3. Computer simulated result for $N=14$. $\varepsilon=2.6, \omega=1.37, \gamma=$ $(0.2$ or -0.2$), \eta=0.01$. Upper figures: $x_{k}$ vs $y_{a k}+y_{b k}$. Middle figures: $x_{k}$ vs $x_{(k+1)}$. Lower figures: $\tau$ vs $x_{k} . k=1,2,3, \ldots, 14$.

\section{B. Odd Number Coupling: $N=15$}

Figure 4 shows the computer simulated result for the case of $N=15$. We can see that the array of oscillators coupled by TVR are synchronized with in-phase (Fig. 4(a)). However, the adjacent oscillators are almost synchronized with anti-phase as shown in Fig. 4(b). Because, the boundary condition is the ring structure, the phase difference between the adjacent oscillators in not around $\pi$. Namely, in this case 15-phase synchronization are observed.

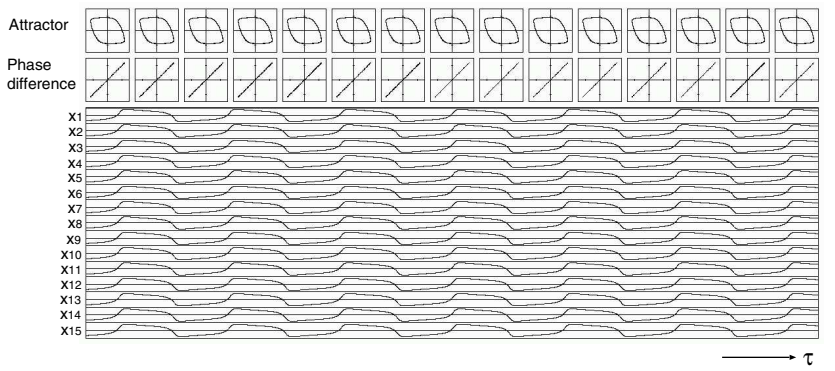

(a) In-phase synchronization.

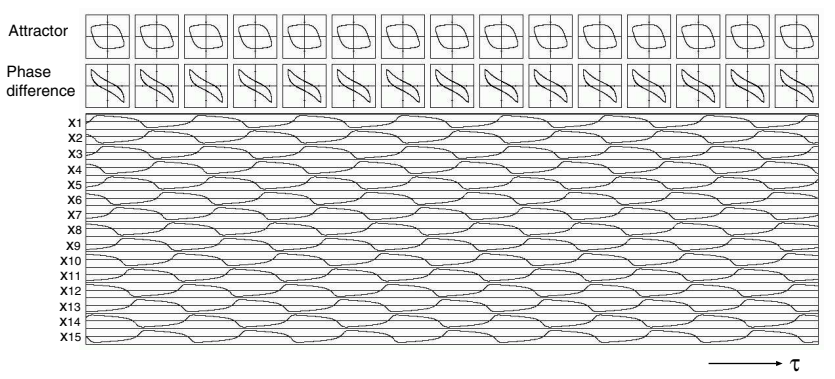

(b) 15-phase synchronization.

Fig. 4. Computer simulated result for $N=15$. $\varepsilon=2.6, \omega=1.37, \gamma=$ $(0.2$ or -0.2$), \eta=0.01$. Upper figures: $x_{k}$ vs $y_{a k}+y_{b k}$. Middle figures: $x_{k}$ vs $x_{(k+1)}$. Lower figures: $\tau$ vs $x_{k} \cdot k=1,2,3, \ldots, 15$.

\section{Coexistence Area}

Next, we investigate coexistence area between in-phase and anti-phase or $N$-phase states when the nonlinearity parameter $\varepsilon$ is changed from 0.4 to 5.0 . We carried out computer calculations for the number of the coupling oscillators is set to $N=15$. The simulated result is shown in Fig. 5. The horizontal axis is $\varepsilon$ and the vertical axis is the frequency of TVR. From this figure, the coexistence range becomes widely with increasing the value of $\varepsilon$. The average value of the coexistence range decreases gradually when $\varepsilon$ increases. We can confirm that the special section around $\varepsilon=4.0$, the coexistence range is narrow extremely.

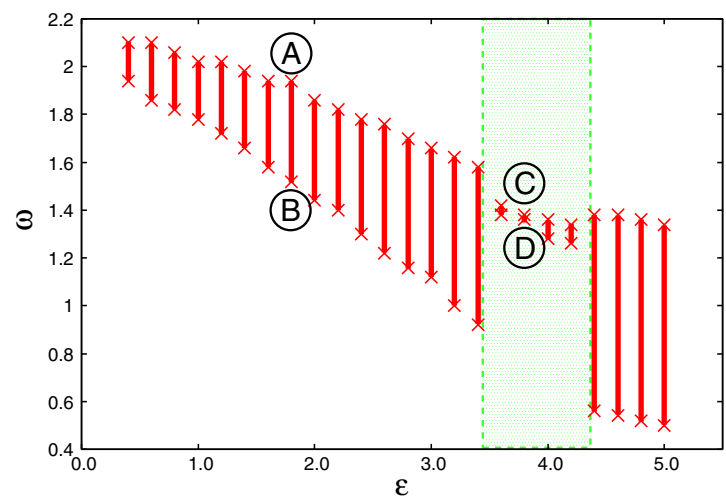

Fig. 5. Coexistence area of in-phase and anti-phase synchronizations. $\gamma=$ $(0.2$ or -0.2$), \eta=0.01$. 


\section{Wave Propagation Phenomena}

In this section, we pay our attention to synchronization phenomena on outside of coexistence area. The simulation results of four-regions (A), B), (C), (D)) from Fig. 5 are shown in Fig. 6. In this figure, the vertical axis is the sum of the voltages of adjacent oscillators and the horizontal axis is time. White regions in the diagram correspond to the states that the sum of the voltages is close to zero, namely the adjacent two oscillators are synchronized at anti-phase. While, black regions correspond to the state the sum of the voltages has large amplitude. We can see that the adjacent two oscillators are synchronized at in-phase in the black regions form Fig. 6. We carried out computer simulation by setting initial condition at random. In the region- $\mathrm{A}$, wave extinction can be observed and the synchronization states becomes stable at in-phase in whole oscillators finally (Fig. 6 (a)). In the region-B, the coupled oscillators produce complex pattern, however the oscillators do not synchronize stably (Fig. 6 (b)). In the region-C, we can confirm the wave propagation phenomena continue to exist (Fig. 6 (c)). In the region-D, the clustering phenomena can be observed (Fig. 6 (d)). We consider that it becomes possible to generate interesting synchronization phenomena by coupling TVR.

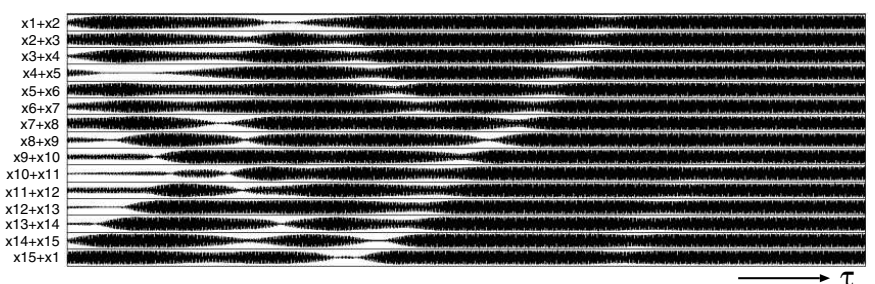

(a) Region-A: $\varepsilon=1.8, \omega=1.99, \gamma=(0.2$ or -0.2$)$ and $\eta=0.01$.

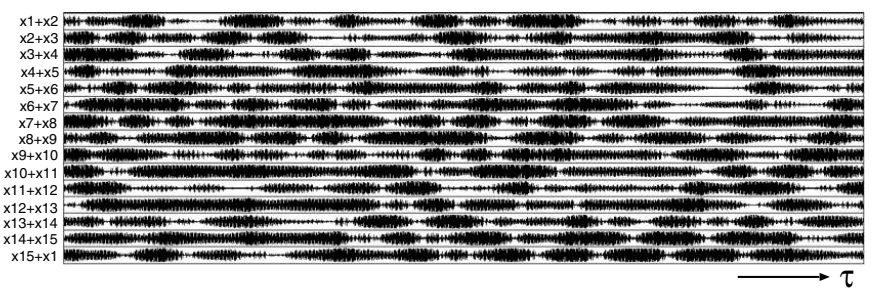

(b) Region-B: $\varepsilon=1.8, \omega=1.47, \gamma=(0.2$ or -0.2$)$ and $\eta=0.01$.

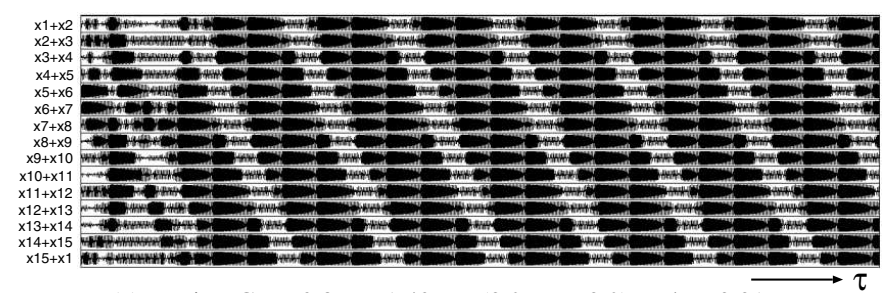

(c) Region-C: $\varepsilon=3.8, \omega=1.43, \gamma=(0.2$ or -0.2$)$ and $\eta=0.01$.

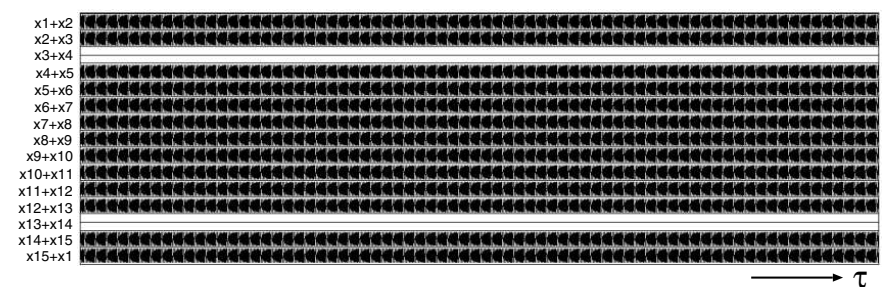

(d) Region-D: $\varepsilon=3.8, \omega=1.31, \gamma=(0.2$ or -0.2$)$ and $\eta=0.01$.

Fig. 6. Synchronization phenomena.

\section{TVR With Timing Mismatch}

We consider the synchronization phenomena when timing mismatch is added to the switching of TVR. The characteristic of TVR with timing mismatch is shown in Fig. 7. From this figure, $T_{s}$ means the one period of TVR and $T_{\varepsilon}$ denotes the maximum value of timing mismatch. We produce the timing mismatch range of 0 to $T_{\varepsilon}$ by uniform random.

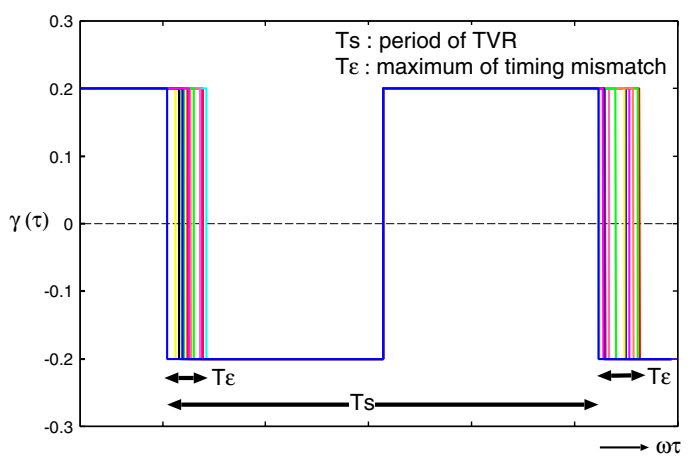

Fig. 7. TVR with timing mismatch $(\mathrm{N}=15)$.

Figure 8 shows the simulation results when the parameters are set to region (A), (B), (C) and (D) from Fig. 5. In the regionA, wave propagation phenomena can be observed (Fig. 8 (a)). In the region- $\mathrm{B}$, the coupled oscillators are synchronized in anti-phase state (Fig. 8 (b)). In the region-C, wave extinction can be observed and the synchronization states becomes inphase (Fig. 8 (c)). In the region-D, the clustering phenomena can be observed (Fig. 8 (d))

From these results of Fig. 6 and 8, we compare the synchronization phenomena between the original TVR and the TVR with timing mismatch. By adding the timing mismatch to the switching of TVR, the different type of synchronization phenomena can be obtained. Namely, in the region-A, the wave extinction change to wave propagation phenomena by adding timing mismatch. In the region- $\mathrm{B}$, the complex pattern disappear and the synchronization state becomes anti-phase. In the region- $\mathrm{C}$, the wave propagation phenomena changes to the wave extinction. Finally, in the region-D, we can confirm the same synchronization as clustering.

Next, we investigate the synchronization phenomena in Region-C when the value of timing mismatch is changed from 0 to 0.25 . The simulated result is shown in Fig. 9. Fist, for smaller $T_{\varepsilon} / T_{s}$, the coupled oscillators produce wave propagation. Second, for intermediate $T_{\varepsilon} / T_{s}$, only in-phase synchronization state can be observed. Finally, for larger $T_{\varepsilon} / T_{s}$, we could not confirm synchronization phenomena, namely the coupled oscillators do not synchronous.

Furthermore, we show synchronization phenomena when the timing mismatch becomes large $\left(T_{\varepsilon} / T_{s}=0.3\right)$ and one example of simulated result is shown in Fig. 10. From this result, we confirm that the coupled oscillators are not synchronized stably. We consider that timing of TVR switching is important role for synchronization state of whole oscillatory systems.

\section{CyCle SWitching of TVR}

Finally, we propose a cycle switching method of TVR as follows. In this method, the switching of TVR is shifted by $2 \pi / N$ regularly as shown in Fig. 11. Figure 12 shows the simulation result. We can confirm that the two types of wave propagation repeat with irregularity. The wave which forms a 


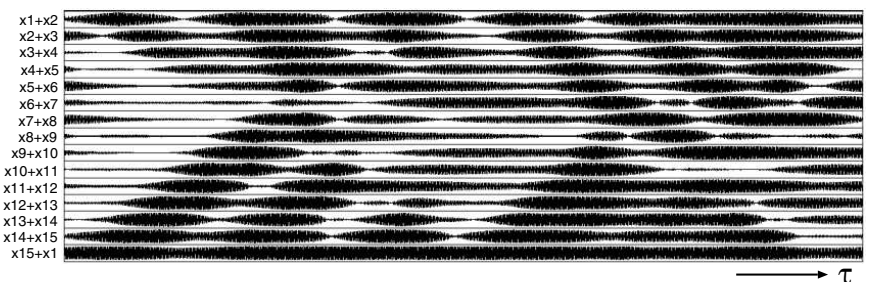

(a) Region-A: $\varepsilon=1.8, \omega=1.99, \gamma=(0.2$ or -0.2$)$ and $\eta=0.01$.

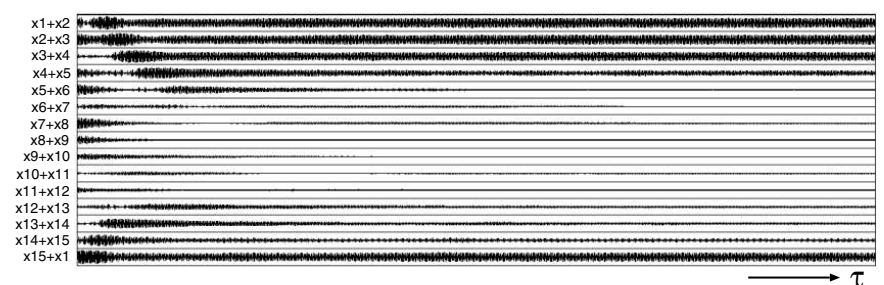

(b) Region-B: $\varepsilon=1.8, \omega=1.47, \gamma=(0.2$ or -0.2$)$ and $\eta=0.01$.

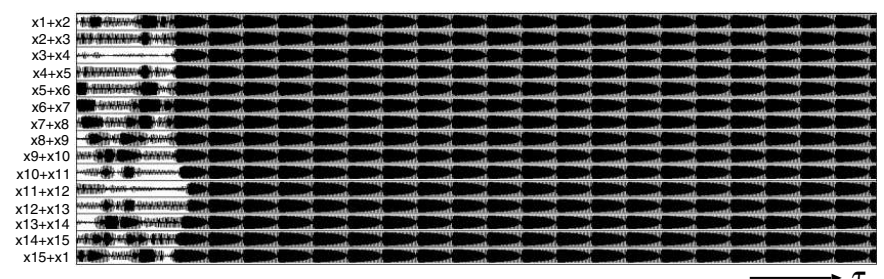

(c) Region-C: $\varepsilon=3.8, \omega=1.43, \gamma=(0.2$ or -0.2$)$ and $\eta=0.01$.

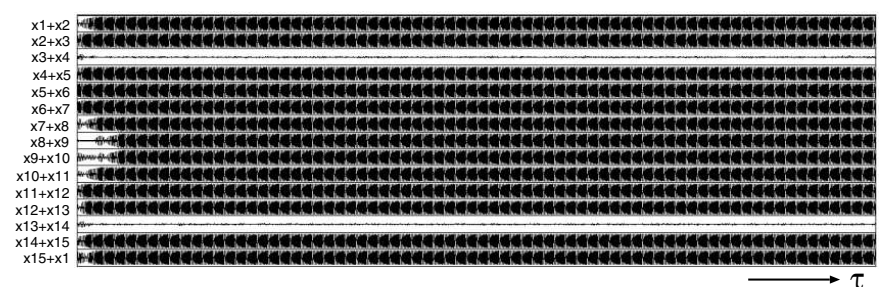

(d) Region-D: $\varepsilon=3.8, \omega=1.31, \gamma=(0.2$ or -0.2$)$ and $\eta=0.01$.

Fig. 8. Synchronization phenomena for TVR with timing mismatch

diamond propagates and the waveform changes to one wave propagation.

\section{CONCLUSIONS}

In this study, we have investigated synchronization phenomena in van der Pol oscillators coupled by a time-varying resistor as a ring. By carrying out computer simulations, we confirm the various interesting phenomena (wave propagation, clustering, complex phase pattern) which cannot be observed in simple oscillatory systems coupled by a resistor.

\section{REFERENCES}

[1] T. Endo and S. Mori, "Mode analysis of a ring of a large number of mutually coupled van der Pol oscillators," IEEE Trans. Circuits Syst., vol.25, no.1, pp.7-18, Jan. 1978.

[2] M. Yamauchi, Y. Nishio and A. Ushida, "Phase-waves in a ladder of oscillators" IEICE Trans. Fundamentals, vol.E86-A, no.4, pp.891-899, Apr. 2003.

[3] Y. Uwate and Y. Nishio, "Complex Phase Synchronization in an Array of Oscillators Coupled by Time-Varying Resistor," Porc. of IJCNN'06, pp. 8345-8350, Jul. 2006.

[4] Y. Nishio and S. Mori, "Mutually coupled oscillators with an extremely large number of steady states," Proc. of ISCAS'92, vol.2, pp.819-822, May 1992.

[5] Y. Nishio and S. Mori, "Chaotic phenomena in nonlinear circuits with time-varying resistors," IEICE Trans. Fundamentals, vol.E76-A, no.3, pp.467-475, Mar. 1993.

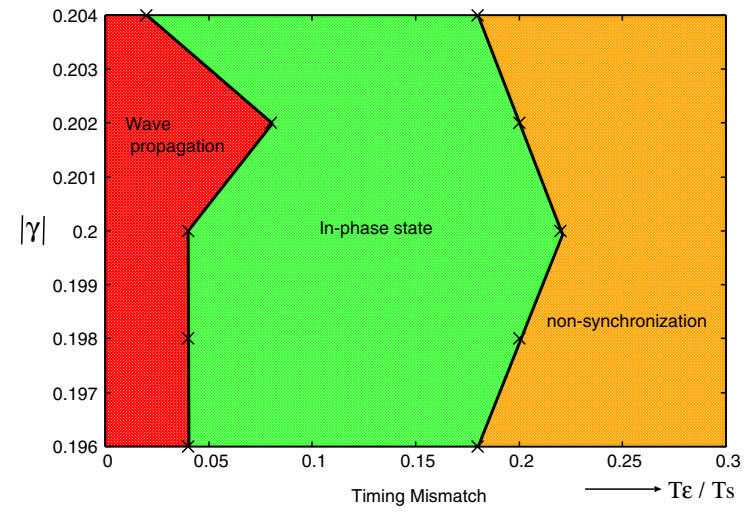

Fig. 9. Synchronization types in Region-C. $\varepsilon=3.8, \omega=1.43, \gamma=(0.2$ or -0.2$)$ and $\eta=0.01$.

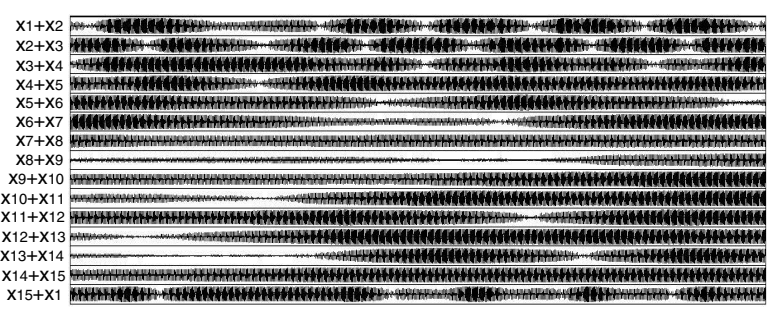

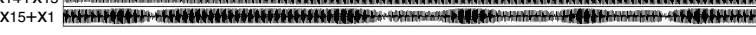

Fig. 10. Synchronization phenomena for TVR with parameter mismatch $\left(T_{\varepsilon} / T_{s}=0.3\right)$. (Region-C: $\varepsilon=3.8, \omega=1.43, \gamma=(0.2$ or -0.2$)$ and $\left.\eta=0.01\right)$.

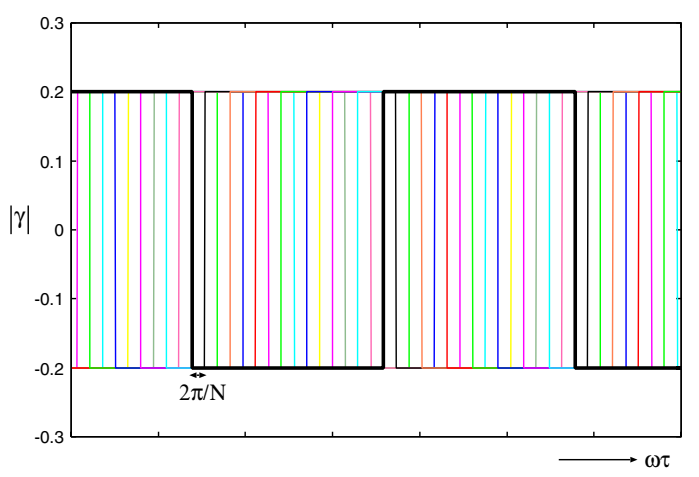

Fig. 11. TVR with cycle switching $(\mathrm{N}=15)$.

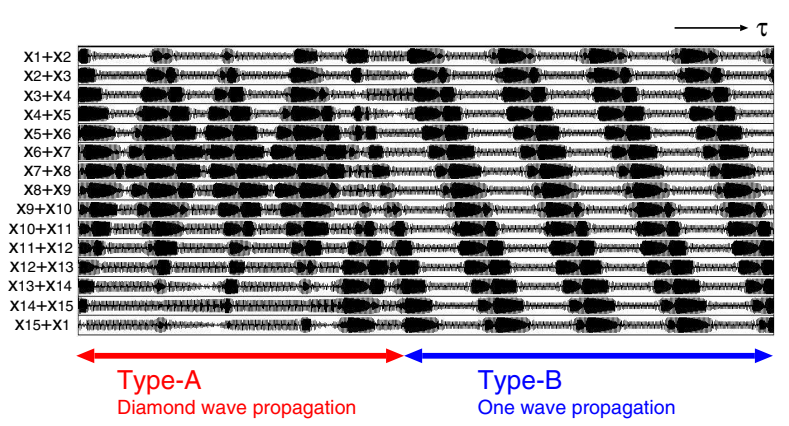

Fig. 12. Two types of wave propagation for TVR with cycle switching. (Region-C: $\varepsilon=3.8, \omega=1.43, \gamma=(0.2$ or -0.2$)$ and $\eta=0.01)$. 